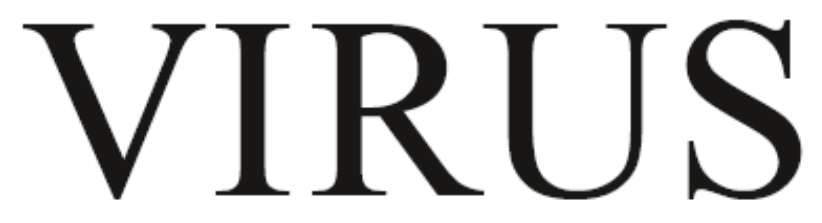

Beiträge zur Sozialgeschichte der Medizin

Band 7

Herausgegeben von

Sonia Horn, Marcel Chahrour und Carlos Watzka

für den Verein für Sozialgeschichte der Medizin

Wien: Verlagshaus der Ärzte, 2008

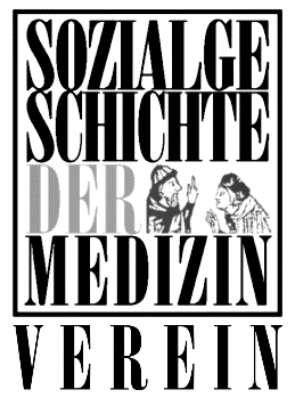


GABRIELE CZARNOWSKI

"RUSSENFETEN"

\section{Abtreibung und Forschung an schwangeren Zwangsarbeiterinnen in der Universitätsfrauenklinik Graz 1943-45'}

In einem Ermittlungsakt der Nachkriegszeit findet sich ein seltenes Dokument, dem ich auch den Titel meines Beitrags entnommen habe. Es handelt sich um die Abschrift aus einem privaten Notizbuch des Dozenten Dr. Franz Hoff (1909-1986), bis 1945 Oberarzt an der Grazer Universitätsfrauenklinik, in welchem er in den Jahren 1942 bis 1944 aus seiner Sicht kritische Vorfälle in der Klinik festgehalten hat. Hoff, geboren im Banat zu k.k.-Zeiten, nach dem Ersten Weltkrieg jugoslawischer Staatsbürger und 1940 in Heidelberg „eingedeutscht“, hatte in Graz studiert, sich in Heidelberg habilitiert und begann Anfang des Jahres 1942 seinen Dienst an der Grazer Universitätsfrauenklinik. ${ }^{2}$ Ich zitiere einige Einträge vom Januar 1944:

„22.1.44. Prof[essor] soll einige Patienten ansehen, angeblich keine Zeit.

Um 7 Russinnen zu untersuchen hat er aber Zeit. (Dr. Bezner)

23.1.44. Der Verbrauch von Röntgenfilmen ist enorm. (Aufnabmen der Russenfeten)!

23.1.44. Um deutsche Frauen kümmerte er sich gar nicht! Wegen Russinnen muss die Operation einer deutschen Frau aufgeschoben werden um 2 Tage.

F. Rosel - Eklampsie [durch Stoffwechselstörungen in der Schwangerschaft ausgelöste Anfälle]: Ob ich sterbe oder nicht Herrn Prof.[essor] so egal (E. war nicht 3 Tage zur Visite erschienen)

26.1.44. Russinnen liegen herum. Kommen nicht zur Interruption dran. - Für deutsche Frauen deshalb kein Platz. Ein grosser Saal muss geräumt werden für die Russinnen.

27.1.44. Peritonitis [Bauchfellentzündung] bei Neo colli III [Gebärmutterhalskrebs im 3. Stadium] noch Radium Einlagen. - E. hat angeblich keine Zeit um nach der Frau zu sehen (II. Kl[asse] !) aber am gleichen Tag werden Russinnen untersucht und behandelt!

28.1.44. Schwestern beklagen sich, dass die Russinnen besser behandelt werden als deutsche Frauen (Oberschwester Sigrid).

29.1.44. Mastitis [Brustdrüsenentzündung] Pat[ientin] (Lehrerin) sagt Ass [istent] Stadler, [...] ob denn die Russin mit der Mastitis auch schon entlassen sei. Es wäre doch nicht recht, dass deutsche Frauen nach Haus müssen, während Russinnen hier bleiben dürfen. "3

In diesen Notizen erscheinen die Verhältnisse auf den Kopf gestellt, die Klassen- oder zutreffender: „Rassen“-Hierarchie in der Klinik umgestülpt. Der Chef bevorzugt „Russinnen“ und vernachlässigt „deutsche
1 Dieser Beitrag entstand im Rahmen des FWF-Projekts „Gynäkologie im Nationalsozialismus. Die Universitätsfrauenklinik Graz 1938-1945“ am Institut für Sozialmedizin und Epidemiologie, Medizinische Universität Graz (Leitung Éva Rásky). Eine Monographie ist in Vorbereitung.

2 Petra SCHEIBLECHNER, „... politisch ist er einwandfrei..." Kurzbiographien der an der Medizinischen Fakultät der Universität Graz in der Zeit von 1938 bis 1945 tätigen WissenschaftlerInnen (= Publikationen aus dem Archiv der Universität Graz 39, Graz 2002) 87-88.

3 Steiermärkisches Landesarchiv (StLA), LG für Strafsachen Graz 19 Vr 20/1947,175-177. 
4 Vgl. zu Zacherl und Ehrhardt mit weiteren Literaturhinweisen: Gabriele CZARNOWSKI, Vom „reichen Material ... einer wissenschaftlichen Arbeitsstätte“. Zum Problem missbräuchlicher medizinischer Praktiken an der Grazer Universitätsfrauenklinik in der Zeit des Nationalsozialismus. In: Wolfgang FREIDL, Werner SAUER (Hg.), NS-Wissenschaft als Vernichtungsinstrument. Rassenhygiene, Zwangssterilisation, Menschenversuche und NS-Euthanasie in der Steiermark (Wien 2004) 225-273, hier 236-237.

5 Bundesarchiv Berlin (BA) (ehem. BDC), SSO, Ehrhardt, Dr. Karl 24.2.1895.

6 Vgl. zum Folgenden ausführlicher CZARNOWSKI, Material 252-258; Ulrike KLEINERT, Radium-Jubel und Röntgen-Wertheim. Gynäkologische Radiologie an der Frankfurter Universitäts-Frauenklinik von den Anfängen bis 1938 (= Frankfurter Beiträge zur Geschichte, Theorie und Ethik der Medizin 8, Hildesheim 1988), 125-128.

7 Vgl. Gabriele CZARNOWSKI, „Die restlose Beherrschung dieser Materie“. Beziehungen zwischen Zwangssterilisation und gynäkologischer Sterilitätsforschung im Nationalsozialismus. In: Zeitschrift für Sexualforschung 14 (2001) 226-246.

8 Karl EHRHARDT, Der trinkende Fötus. Eine röntgenologische Studie. In: Münchener medizinische Wochenschrift 84 (1937) 1699-1700; Karl EHRHARDT, Atmet das Kind im Mutterleib? Eine röntgenologischen Studie. In: Münchener medizinische Wochenschrift 86 (1939) 915-918.

9 Karl EHRHARDT, Weitere Erfahrungen mit meiner Methode der intraamnialen Thoriuminjektion. Fetale Organographie. In: Zentralblatt für Gynäkologie 65 (1941) 114-120, hier 119.
Frauen“ - das ist die Ansicht von Oberarzt und Assistenzarzt/ärztin, Oberschwester und Schwestern, auch von „Klasse“-Patientinnen, die sich normalerweise der besonderen Gunst des Klinikleiters erfreuen. Dessen ganze Aufmerksamkeit gelte aber den „Russinnen“: Sie nehmen auf sein Geheiss den „Deutschen“ (gemeint waren Österreicherinnen) Raum und Zeit weg, sie „liegen herum“, und die „Interruption“ [Schwangerschaftsabbruch], wegen der sie eingeliefert worden sind, wird hinausgezögert. Warum? Die Abbrüche als solche hingegen erscheinen nicht als Ärgernis.

I.

„E.“, der „Prof.“, das war Karl Ehrhardt (1895-1993), der 1939 aus politischen Gründen mit Rückenwind des „Reichsgesundheitsführers“ Leonardo Conti und - wie es in mehreren Quellen kolportiert wird - auch mit Unterstützung der SS auf die Grazer Lehrkanzel berufen wurde, die seit der politisch motivierten Entlassung Hans Zacherls (1889-1968) im August 1938 vakant gewesen war. ${ }^{4}$ Ehrhardt, NSDAP- und SS-Mitglied seit 1933 sowie anlässlich seiner Bestellung zum Grazer Klinikchef - mit Überspringen gleich zweier Dienstgrade - vom SS-Untersturmführer zum SS-Sturmbannführer befördert ${ }^{5}$, kam aus der Seitz'schen Klinik in Frankfurt am Main. Er hatte sich 1931 habilitiert und galt als Spezialist auf dem Gebiet der Endokrinologie. Ein anderer Forschungsschwerpunkt Ehrhardts waren die „Placentographie“ und die „Fetographie“. Hierbei handelte es sich um die röntgenologische Darstellung der Plazenta bzw. tierischer und menschlicher Leibesfrüchte nach der Injektion eines Röntgenkontrastmittels in den mütterlichen Leib. ${ }^{6}$ Experimente zur Entwicklung intrauteriner bildgebender Verfahren hatte Ehrhardt seit 1931 durchgeführt, zunächst an der Universitätsfrauenklinik in Frankfurt am Main, dann als Klinikchef in Graz. Seine menschlichen Forschungsobjekte waren Anfang der 30er Jahre Patientinnen, die zum Schwangerschaftsabbruch aus medizinischen Gründen in die Frankfurter Universitätsfrauenklinik (UFK) überwiesen worden waren. Nach Beginn der nationalsozialistischen Zwangssterilisationen und den daran gekoppelten eugenischen Abtreibungen missbrauchte er auch schwangere Sterilisandinnen für seine wissenschaftlichen Interessen. Sie vergrößerten den „Patientinnenpool“, aus dem er sich für seine Forschungen bediente, erheblich; doch er war nicht der einzige deutsche Gynäkologe, der an zur Zwangssterilisation (mit oder ohne Abtreibung) eingewiesenen Patientinnen Versuche anstellte. ${ }^{7}$

Ehrhardts Forschungen zur Fetographie wurden von der Deutschen Forschungsgemeinschaft finanziell gefördert und führten zu mehreren Publikationen, u.a. „Der trinkende Fötus. Eine röntgenologische Studie“ (1937) und „Atmet der Fötus im Mutterleib?“ mit demselben Untertitel (1939). ${ }^{8} 1941$ folgten - nun aus Graz - „Weitere Erfahrungen mit meiner Methode der intraamnialen Thoriuminjektion. Fetale Organographie“. Abgebildet im Zentralblatt für Gynäkologie sind 
Röntgenaufnahmen von fünf Leibesfrüchten im dritten bis sechsten Schwangerschaftsmonat „in natürlicher Grösse“, die (bis auf eine Abbildung aus Frankfurt) eine Auswahl aus den „zahlreichen Röntgenbildern“ darstellte, die - so der Verfasser - „ich im Laufe der letzten zwei Jahre mit meiner Methode [...] erzielen konnte". ${ }^{9}$ Neu gegenüber seinen früheren Versuchen waren u.a. die Verwendung eines aggressiveren Kontrastmittels sowie die Erhöhung der Zahl der Röntgenaufnahmen an der jeweiligen schwangeren Frau nach der Injektion. Damit verlängerte sich der Zeitraum vom Beginn der Experimente bis zur Vornahme der Abtreibung (und Zwangssterilisation) um mehrere Tage. Beteiligt war auch ein Assistenzarzt der Frauenklinik, Dr. Richard Bayer (1907-1989). Er hatte die Wirkung des aggressiveren Konstrastmittels an „zwei nicht lebensfähigen [aber noch lebenden, GC] frühgeborenen Früchten aus dem 7. Schwangerschaftsmonat" untersucht. Über die Herkunft dieser Frühgeburten ist nichts weiteres bekannt. ${ }^{10}$ Ausserdem kooperierten bei Untersuchungen an toten Leibesfrüchten mit den Mitteln und Fragestellungen ihrer Fachgebiete Prof. Dr. Alfred Pischinger (1899-1983), Vorstand des Universitätsinstituts für Histologie und Embryologie, ${ }^{11}$ und Prof. Dr. Georg Gorbach (1901-1970) vom Institut für Biochemie und Mikrobiologie der Technischen Hochschule Graz. ${ }^{12}$

Am Ende von Ehrhardts röntgenologischen Versuchen mit schwangeren Frauen stand in der Regel der Abbruch der Schwangerschaft. ${ }^{13}$ Auch die Wahl der Abtreibungsmethode richtete er nicht nach dem Wohl der Patientin, sondern an seinen Forschungszielen aus. Weil er die Leibesfrüchte möglichst unverletzt in ihrer Eihülle „gewinnen“ wollte, operierte er bereits in frühen Schwangerschaftsstadien nach Verfahren, die dem zeitgenössischen Standard ärztlicher Kunst für Abbrüche in diesem Zeitraum nicht entsprachen. An Stelle der üblichen Curettage wählte die eingreifendere „Sectio parva“ und die „Hysterotomia vaginalis anterior".

Ehrhardt war damals nicht der einzige „Fetograph“ in Deutschland, aber nahm für sich in Anspruch, der erste gewesen zu sein. Zwei Ärzte der UFK Würzburg probierten 1937 seine Methode an zwei Patientinnen aus, die zum Schwangerschaftsabbruch aus medizinischer Indikation in die Klinik überwiesen worden waren. ${ }^{14}$ Im klinischen Wörterbuch Pschyrembel von $1977^{15}$ ist die Fetographie mit dem Gynäkologen Joachim Erbslöh und der Jahreszahl 1935 verbunden; Erbslöh (1909-2006) forschte an der medizinischen Akademie in Danzig und ab 1939 im besetzten Bydgoszcz/Bromberg (Polen). ${ }^{16}$

Mit der Berufung Ehrhardts an die Grazer UFK traf ein Vertreter der operativ konservativen, in der Krebs- und Myombehandlung die Strahlentherapie bevorzugende gynäkologischen Schule von Ludwig Seitz ${ }^{17}$ auf Oberärzte und Assistenten, die in der Tradition der operativ aktiven Wiener gynäkologischen Schule Wertheims und Schautas standen. Sie waren ihm als Operateure überlegen, nicht nur bei Krebsoperationen, sondern auch in der Geburtshilfe, etwa bei Indikationen für die
10 Alfred PISCHINGER, Über das Wesen kindlicher Atembewegungen vor der Geburt. In: Zentralblatt für Gynäkologie 65 (1941) 120-124, hier 123. $\mathrm{Zu}$ Bayer vgl. SCHEIBLECHNER, Kurzbiographien 6-8.

11 PISCHINGER, Atembewegungen. Hinsichtlich der Involvierung $\mathrm{Pi}$ schingers in die nationalsozialistische Eugenik und seiner Einstellung zum Krankenmord in jenen Jahren soll erwähnt werden, dass er nebenberuflich „Erbgesundheitsrichter" am Grazer Erbgesundheitsobergericht (EOG) war und dass er sich in einer seiner Vorlesungen „öffentlich rühmte, seinen gehirngeschädigten Sohn Klaus der ,Euthanasie' übergeben zu haben ": Gerhard HARTMANN, Im Gestern bewährt - Im Heute bereit. 100 Jahre Carolina. Zur Geschichte des Verbandskatholizismus (= Grazer Beiträge zur Theologiegeschichte und Kirchlichen Zeitgeschichte 2, Graz Wien Köln 1988) 404. Die EOG entschieden in zweiter und letzter Instanz über Zwangssterilisationen nach dem "Gesetz zur Verhütung erbkranken Nachwuchses“. Vgl. Birgit POIER, „Erbbiologisch unerwünscht“. Die Umsetzung rassenhygienisch motivierter Gesundheits- und Sozialpolitik in der Steiermark 1928-1945. In: Freidl/Sauer, NS-Wissenschaft 177-224, hier 211, mit dem aus einer Quelle entnommenen nicht zutreffenden Vornamen Arnold. Vgl. auch SCHEIBLECHNER, Kurzbiographien 192-194.

12 Diese Kooperation geht hervor aus: PISCHINGER, Atembewegungen 123. Ich danke Dr. Marieluise Vesulak für die Mitteilung von Vornamen und Lebensdaten Prof. Gorbachs.

13 Nach eigenen Angaben wandte er zweimal seine Methode als diagnostisches Mittel zur Überprüfung des vermuteten Todes der Leibesfrucht an, was sich in nur einem Fall bestätigte. Im zweiten Fall - es handelte sich um „ein 17-jähriges unverheirates Mädchen" - kam es angeblich zu keiner Schädigung des Kindes. Vgl. EHRHARDT, Atmet das Kind 917.

14 Vgl. CZARNOWSKI, Material 254, Fn 102.

15 Willibald PSCHYREMBEL, Klinisches Wörterbuch, 253. Auflage (Berlin / New York 1977) 361.

16 Vgl. Joachim ERBSLÖH, Das intrauterine Fetogramm. In: Archiv für Gynäkologie 174 (1942) 160-162 (= Verhandlungen der deutschen Gesellschaft für Gynäkologie 1941).

17 Vgl. KLEINERT, Radium-Jubel; Wolfgang FROBENIUS, Röntgenstrahlen 
statt Skalpell. Die Erlanger Frauenklinik und die Geschichte der Radiologie 1914-1945 (= Erlanger Forschungen Reihe B 26, Erlangen 2003).

18 Vgl. Martina SCHLÜNDER, Graz 1939: Die Konsequenzen einer Fehlbesetzung. Unveröffentlichtes Manuskript, Berlin 1998. Vgl. zum folgenden ausführlicher CZARNOWSKI, Material 237-238.

19 Universitätsarchiv der Karl FranzensUniversität Graz (UAG), Personalakt Prof Dr. Karl Ehrhardt, Bericht Prof. Dr. Wagner vom 12.6.1942. In CZARNOWSKI, Material, habe ich den Personalakt fälschlicherweise als Disziplinarakt bezeichnet.

20 Zur Zwangsarbeit in Österreich während des II. Weltkriegs mit Angaben auch über die Steiermark vgl. Florian FREUND, Bertrand PERZ, Die Zahlenentwicklung der ausländischen Zwangsarbeiter und Zwangsarbeiterinnen auf dem Gebiet der Republik Österreich 1939-1945. Gutachten im Auftrag der Historikerkommission der Republik Österreich, Wissenschaftliche Redaktion: Eva BLIMLINGER (Wien 2000) (http:// www.historikerkommission.gv.at/ deutsch_home.html / Ergebnisberichte); ÖSTERREICHISCHE HISTORIKERKOMMISSION (Hg.), Zwangsarbeiter und Zwangsarbeiterinnen auf dem Gebiet der Republik Österreich 1949-1945. Mit Beiträgen von Mark SPOERER, Florian FREUND, Bertrand PERZ (Zwangsarbeit auf dem Gebiet der Republik Österreich 1, München 2004); Stefan KARNER, Peter RUGGENTHALER, Zwangsarbeit in der Land- und Forstwirtschaft auf dem Gebiet Österreichs 19391945 (Zwangsarbeit auf dem Gebiet der Republik Österreich 2, München 2004); Ela HORNUNG, Ernst LANGTHALER, Sabine SCHWEIZER, Zwangsarbeit in der Landwirtschaft in Niederösterreich und dem nördlichen Burgenland (Zwangsarbeit auf dem Gebiet der Republik Österreich 3, München 2004).

21 Vgl. Gabriele CZARNOWSKI, Women's crimes, state crimes: abortion in Nazi Germany. In: Margaret L. ARNOT, Cornelie USBORNE (Hg.), Gender and Crime in Modern Europe (London 1999) 238-256.
Anwendung der Zange. Binnen kurzem kam es zwischen den österreichischen Assistenten und ihrem neuen „reichsdeutschen“ Chef zu dem, was Martina Schlünder als „Schulen- und Mentalitätenstreit“ beschrieben hat. ${ }^{18}$ Nicht nur aus der Sicht seiner Assistenten beherrschte Ehrhardt die großen Operationen der Wiener Schule unzureichend. Zwei Kommissionen untersuchten kursierende Gerüchte und Vorwürfe über geburtshilfliche und gynäkologische Kunstfehler, darunter auch sieben Todesfälle. Von beiden Kommissionen wurde er - mit massiver Unterstützung der Fakultät - rehabilitiert. Erfolgte die erste Untersuchung 1940 noch universitätsintern, rückte Ende 1941 eine von der Wissenschaftsabteilung des Reichserziehungsministeriums in Berlin bestimmte Kommission dreier Ordinarien (Georg August Wagner aus Berlin, Carl Josef Gauss aus Würzburg, Ernst Philipp aus Kiel) in Graz an. Ihr Bericht vom Juli 1942 konzedierte, es sei „keine Frage, dass dem Prof. Ehrhardt Vorfälle beim Operieren unterlaufen sind". Es sei ihm aber anzurechnen, dass er seine operative Insuffizienz für Graz sehr bald eingesehen und „,...] durch fleissiges Operieren an der Leiche, durch wiederholte Reisen zu anerkannten Operateuren, besonders auch der Wiener Schule [...] viel dazu gelernt" habe, er scheine sich „namentlich nach den operativen Resultaten des letzten Jahres [...] wesentlich vervollkommnet und so zumindest das Niveau erreicht zu haben, das manche gewesene und jetzige Direktoren von Universitätsfrauenkliniken in operativer Beziehung einnehmen. " ${ }^{19}$ Hoffs Aufzeichnungen aus dem Jahr 1944 dokumentieren allerdings, dass Ehrhardt noch immer gefährliche Missgriffe und Fehler bei Operationen und Geburten unterliefen und dass er sich „an schwierige Fälle nicht heran[traute]“. Auch wenn der Klinikchef siegreich aus beiden Untersuchungen hervorgegangen war und die ihn kritisierenden Assistenzärzte gemassregelt wurden und die Klinik verlassen sollten, so resultierte doch aus diesen Vorkommnissen eine Dynamik, in der Ehrhardt vom „Operieren an der Leiche“ fortschritt zu Operationsübungen an gesunden, lebendigen Menschen, die im „Dritten Reich“ als „Untermenschen“ galten.

II.

„Die Russinnen“ - damit bezeichnete Dr. Hoff die in die Grazer UFK zum Schwangerschaftsabbruch eingewiesenen Zwangsarbeiterinnen aus den Ländern der damaligen Sowjetunion und aus Polen. ${ }^{20}$ Grundlage dieser Abbrüche war ein Erlass des „Reichsgesundheitsführers“ Dr. Conti vom März 1943. Er wurde wenige Tage nach einer Änderung der Abtreibungsparagraphen zum internen Gebrauch weitergegeben. Diese nationalsozialistische Reform sah parallel zur Einführung der Todesstrafe für Lohnabtreibung an „deutschen“ Frauen die Aufhebung der polizeilichen und gerichtlichen Verfolgung von Abtreibungen an nichtdeutschen Frauen vor. ${ }^{21}$ Waren bis Ende 1942 schwangere Zwangsarbeiterinnen noch in ihre Herkunftsländer abgeschoben worden, so organisierte die Gesundheitsverwaltung nun in Kooperation 
mit der Himmlerbehörde „Reichskommissar für die Festigung deutschen Volkstums“ und der Reichsärztekammer Schwangerschaftsabbrüche an „Ostarbeiterinnen“ in grossem Massstab. Eine Rückkehr in die Heimat wurde ausgeschlossen. Dem Gebot, möglichst zahlreich an ihnen abzutreiben, lag neben der vollen Ausnützung ihrer Arbeitskraft die Vorbeugung „volkstumspolitischer Gefahren“ zugrunde, die mit der Geburt „fremdvölkischer“ Kinder befürchtet wurde. ${ }^{22}$

Die Kontrolle über die Abbrüche bei „Ostarbeiterinnen“ und Polinnen oblag den „Gutachterstellen für Schwangerschaftsunterbrechung aus gesundheitlichen Gründen“ der Ärztekammer, derselben Institution, die auch für die Entscheidung medizinisch indizierter Abtreibungen bei „deutschen“ Frauen zuständig war. Während sie bei diesen im Sinne der Geburtenförderung äusserst restriktiv entschied, wurden die Anträge für den Abbruch der Schwangerschaft bei „Ostarbeiterinnen“ und Polinnen generell „genehmigt“, es sei denn, ein „germanischer Erzeuger" war im Spiel oder - sofern es sich um Polinnen handelte - der Gutachter beurteilte sie als „gutrassisch“. Dann wurde die Frau zur „Rassenprüfung“ durch einen „Eignungsprüfer“ des SS-Rassen- und Siedlungshauptamts ins Gesundheitsamt vorgeladen und die Abtreibung möglicherweise verboten, um das Kind dem „deutschen Volkskörper“ einzuverleiben. ${ }^{23}$ So etwas kam selten vor. Formal war die „Einwilligung“ der Frauen zum Abbruch nötig, der ihnen aus „sozialen“ Gründen schmackhaft gemacht werden sollte. Die Erwähnung rassischer Gründe war strikt verboten. Die Frauen wurden zur Unterschrift gedrängt, genötigt oder gezwungen, und wenn es nicht - wie häufig - durch den Arbeitgeber, den Lagerleiter, das Arbeitsamt, die Polizei oder den Amtsarzt geschah, so durch die Not ihrer Lebensumstände. Orte der Abtreibung waren Krankenreviere in Wohnlagern grosser Firmen, Durchgangslager der Arbeitsämter, „Ostarbeiterbaracken “ von Spitälern, aber auch Universitätskliniken. ${ }^{24}$

Die meisten „Russinnen“ in der UFK Graz waren Ukrainerinnen, daneben gab es eine kleinere Anzahl polnischer und russischer Patientinnen. Der erste Nachweis einer solchen Abtreibung in der UFK Graz stammt vom April 1943. Die 22-jährige verheiratete Paulina B. - „Wohnort: St. Peter Ziegelfabrik“, „Beschäftigung: Ostarbeiterin“, wie es im Ambulanzbuch vom 6. April 1943 verzeichnet ist - wurde von Dr. Wilhelm Neugebauer, Distriktsarzt St. Peter bei Graz, überwiesen. Er bat „[...] um Feststellung, ob mit Sicherheit eine Schwangerschaft vorliegt. Im zutreffenden Falle (sei) sie mit einer Unterbrechung, die auf kurzem Wege (telefon[ischer] Anruf bei mir und nachträgliche Übersendung des Antragsformulars) genehmigt werden kann, einverstanden". ${ }^{25}$ Der in der Ambulanz an diesem Tag diensttuende Assistenzarzt Dr. Hans Stadler fand den „Uterus faustgro $\beta$, aufgelockert, antefl[ektiert], bewegl[ich]“, die „Adnexe frei“ und diagnostizierte eine Gravidität im dritten Schwangerschaftsmonat. Die Klinik entschied sich gegen den „kurzen Weg“. Erst eine knappe Woche später wird Paulina B. aufgenommen und nach 13 Tagen, am 24. April 1943, entlassen. Laut
$22 \mathrm{Zu}$ Abtreibungen an Zwangsarbeiterinnen in Wien und in "Oberdonau“ vgl. Herwig CZECH, Zwangsarbeit, Medizin und „Rassenpolitik“ in Wien: Ausländische Arbeitskräfte zwischen Ausbeutung und rassistischer Verfolgung. In: Andreas FREWER, Günther SIEDBÜRGER (Hg.), Medizin und Zwangsarbeit im Nationalsozialismus. Einsatz und Behandlung von „Ausländern“ im Gesundheitswesen (Frankfurt a.M. / New York 2004) 253-280, hier 269-273; Gabriella HAUCH, Zwangsarbeiterinnen und ihre Kinder: Zum Geschlecht der Zwangsarbeit. In: Christian GONSA, Gabriella HAUCH, Michael JOHN, Josef MOSER, Bertrand PERZ, Oliver RATHKOLB, Michaela C. SCHOBER, Zwangsarbeit - Sklavenarbeit: Politik-, sozial- und wirtschaftshistorische Studien (= Oliver RATHKOLB (Hg.), NS-Zwangsarbeit: Der Standort Linz der Reichswerke Hermann Göring AG Berlin, 1938-1945 1, Wien/ Köln/ Weimar 2001) 355-448, hier 422-432.

23 Vgl. allgemein und zu „Rassenprüfungen" im Rheinland: Gisela BOCK, Zwangssterilisation im Nationalsozialismus. Studien zur Frauenpolitik und Rassenpolitik (Opladen 1986) 437-451.

$24 \mathrm{Zu}$ Abtreibungen an Zwangsarbeiterinnen in Universitätsfrauenkliniken - auch zur Ablehnung dieser Eingriffe in Innsbruck, München und Tübingen - vgl. CZARNOWSKI, Material 242-245 mit weiteren Literaturhinweisen; Wolfgang FROBENIUS, Abtreibungen bei "Ostarbeiterinnen “ in Erlangen. Hochschulmediziner als Helfershelfer des NS-Regimes. In: FREWER/ SIEDBÜRGER, Medizin und Zwangsarbeit 283-307.

25 StLA, Frauenklinik Gebärhaus, Karton 113/1943, Zl 1752. 
Eintrag im Standesprotokoll fand der „Abortus“ am 17. April 1943 statt. ${ }^{26}$ Im Mai folgen weitere Abtreibungen dieser Art, zwölf an der Zahl, im Juni neun, im Juli eine. Bis Ende November 1943 waren es dann durchschnittlich 10 pro Monat, im Dezember 21. Im Jahr 1944 nahmen die Schwangerschaftsabbrüche deutlich zu: 26 im Januar, 35 im Februar, 47 in März, 41 im April, 48 im Mai. Der Höhepunkt ist mit 50 Aufnahmen im Juni 1944 erreicht. Allein am 13. und am 19. Juni 1944 wurden jeweils acht schwangere „Ostarbeiterinnen“ „zur Interruptio“ eingewiesen. Zeitweilig war die Gebärklinik überfüllt und Neuaufnahmen mussten zurückgestellt werden. In den nächsten drei Monaten waren es jeweils rund 30 Osteuropäerinnen, die wegen Abtreibung zur Aufnahme kamen; im Oktober 1944 waren es 24. Vom 2. bis zum 16. November wurde an 15 Frauen aus "volkstumspolitischen " Gründen abgetrieben.

Dann trat eine Pause ein, die bis zum 3. Januar 1945 reichte. Der Grund dafür war ein Erlass des Reichsstatthalters an alle Gaukrankenhäuser (ohne Untersteiermark), der die Orte für "Schwangerschaftsunterbrechungen an Ostarbeiterinnen " in der Steiermark neu bestimmte. Diese Abtreibungen sollten künftig nur noch in Lagern durchgeführt werden, mit Ausnahme des Gaukrankenhauses (GKH) in Wagna (für die Kreise Leibnitz und Radkersburg) sowie der „Baracken“ der GKH in Rottenmann (für Liezen) und in Hartberg (für Hartberg, Fürstenfeld und Oberwart). „Alle übrigen Gaukrankenhäuser einschließlich des Gaukrankenhauses Graz-Ost" - damit auch die UFK - hätten „die Aufnahme von schwangeren Ostarbeiterinnen zur Schwangerschaftsunterbrechung abzulehnen und die Ostarbeiterinnen an die zuständige Stelle zu verweisen ". ${ }^{27}$ Das waren für Stadt und Landkreis Graz sowie die Kreise Deutschlandsberg, Feldbach, Voitsberg und Weiz die Lager Liebenau und Steinfeld, ${ }^{28}$ für die Kreise Bruck an der Mur und Mürzzuschlag das Lager Deuchendorf, für Judenburg, Murau und Leoben das Lager Trofaiach, im Kreis Leoben ausserdem das „Lager 64“ in Eisenerz, und für Liezen das „Gemeinschaftslager Schmidhütte“ in Liezen. Zunächst wurde der Erlass an der UFK Graz eingehalten. So findet sich in den Ambulanzunterlagen über die 19-jährige Schura K., im sechsten Monat schwanger und aus der Nähe von Weiz von Dr. Gawalowski „z[ur] Interruptio “ an die UFK überwiesen, für den 21.11.1944 die Bemerkung „Lager Liebenau geschickt“. ${ }^{29}$

StLA, LKH Standesprotokoll Frauen 1943/1-1433.

27 UAG Med. Fak. 1944/45-1603.

28 Vgl. Barbara STELZL, Lager in Graz. Zur Unterbringung ausländischer Zivilarbeiter, Kriegsgefangener und KL-Häftlinge 1938-1945. In: Stefan KARNER (Hg.), Graz in der NSZeit. Begleitband zur Ausstellung (Graz 1998) 353-369, hier 357-358, 360-361. Die Funktion dieser Lager auch als Abtreibungsstätten war bisher nicht bekannt.

29 StLA, Frauenklinik Gebärhaus, Karton 123/1944 Zl. 6520 .

Doch der Klinikvorstand war mit dem Verbot nicht einverstanden. Er intervenierte beim Dekan, dem Anatomen Prof. Dr. Anton Hafferl, und setzte sich mit dem Argument der mangelhaften Versorgung mit Schwangeren im „Lehrbetrieb“, bei ärztlichen Prüfungen und in der Hebammen-Lehranstalt für die erneute Einweisung der zur Interruptio bestimmten „schwangeren Ostarbeiterinnen“ ein: „Da es sich bei den betreffenden Arbeiterinnen nicht nur um Schwangerschaften der ersten zwei Monate, sondern auch um Schwangerschaften der Monate 3, 4, 5 und 6 handelt, wären die [...] Schwierigkeiten durch Aufhebung des Erlasses weitgehendst gemindert". Aus einer Notiz des Dekans über 
ein Gespräch mit dem zuständigen Regierungsdirektor der Gesundheitsbehörde beim Reichsstatthalter, DDr. Julius Strenger, geht hervor, dass dieser „gegen die Verwendung normaler Geburten auf der Klinik soweit sie für den Unterricht notwendig sind, nichts einzuwenden" habe. „Ostarbeiterinnen [dürften] aber nicht mehr im Gaukrankenhaus abortieren".${ }^{30}$ Offenbar konnte Ehrhardt es aber beim Verwaltungsdirektor des GKH erreichen, dass genau dies in seiner Klinik doch wieder praktiziert werden konnte. ${ }^{31}$ Für den Zeitraum vom 3. Januar 1945 bis zum Kriegsende sind weitere 22 eindeutig identifizierbare Schwangerschaftsabbrüche und 39 „Aborte“ von Zwangsarbeiterinnen ebendort belegt.

Im Verlauf des Ermittlungsverfahrens gegen Ehrhardt nach 1945 liess der Untersuchungsrichter eine Liste über nicht von Ehrhardt vorgenommene Abbrüche an „Ostarbeiterinnen“ und Polinnen zwischen September 1943 und März 1945 zusammenstellen. Aus dieser Liste mit gut 300 Eingriffen geht hervor, dass Dr. Hoff 200, der polnisch-ukrainische Arzt Dr. Pruc 10, der spanische Arzt Dr. Martin 55 und weitere fünf Assistenten der Klinik - Dr. Tritthart, Dr. Stadler, Dr. Schneider, Dr. Pöschl sowie die reichsdeutsche Ärztin Dr. Bezner - jeweils zwischen 3 und 13 Abtreibungen durchgeführt hatten. ${ }^{32}$ Die Krankengeschichten, aus denen diese Zahlen eruiert wurden, sind heute nicht mehr vorhanden. Zur Rekonstruktion der Abtreibungen an Zwangsarbeiterinnen habe ich ersatzweise die vollständig überlieferten Personenstandsprotokolle des GKH/LKH und die bis Ende November 1944 überlieferten Ambulanzbücher der Frauenklinik herangezogen. Aus ihnen lässt sich eine ungefähre Summe von 650 erschliessen. Eindeutig identifizierbar sind nach dem derzeitigen Forschungsstand 500 Schwangerschaftsabbrüche an Frauen aus der Ukraine, Russland und Polen. Hinzu kommen 124 „Aborte“, worunter rassisch motivierte Abtreibungen ebenso fallen können wie Fehlgeburten. Auch hinter Standesprotokolleinträgen wie „Grav[idität] $M L$ [= Lunarmonat; Schwangerschaftsmonat] III-IV geh[eilt] “ oder „Grav[idität] geh[eilt]“ verbergen sich teilweise Abtreibungen an „Ostarbeiterinnen“, desgleichen bei den verzeichneten Totgeburten. Von den insgesamt geschätzten 650 Eingriffen gehen die nicht in der besagten Liste von 300 Abtreibungen verzeichneten Eingriffe auf das Konto des Klinikvorstands: Ehrhardt führte mindestens 200, möglicherweise bis zu 350 dieser Schwangerschaftsabbrüche durch. Für denselben Zeitraum sind 220 Entbindungen von Zwangsarbeiterinnen in den Standesprotokollen verzeichnet.

III.

An der UFK Graz hatten Frauen und Mädchen aus den Ländern der damaligen Sowjetunion und Polen medizinische Eingriffe zu erdulden, die über den rassisch motivierten Schwangerschaftsabbruch weit hinaus gingen. Vor allem der Klinikvorstand missbrauchte diese Patientinnen zu eigenen Forschungs- und Übungszwecken. Allein schon seine
30 UAG Med. Fak. 1944/45-1603, 1.12.1944. Hintergrund des „Engpasses" waren laut Ehrhardt Weisungen der Luftschutzbehörde, ,unkomplizierte Geburten und gesunde Schwangere" möglichst nicht mehr aufzunehmen, was er bereits im Februar 1944 hinsichtlich der negativen Folgen für die Lehre moniert hatte. Vgl. UAG Med. Fak. 1943/44-2218.

$31 \mathrm{Vgl}$. dazu und zum Prozedere in der Klinik die Wiedergaben der Nachkriegsaussagen Dr. Hoffs in: CZARNOWSKI, Material 249, 245-246.

32 StLA LG für Strafsachen Graz, Vr 4434/49, Bd. 3. Zu Pruc (alternative Schreibweise: Pruz), Martin, Tritthart, Stadler, Schneider, Pöschl und Bezner vgl. auch SCHEIBLECHNER, Kurzbiographien 201, 161, 259, 245, 227f., 196-198, 15. 
Abtreibungsmethoden wiesen grausame Besonderheiten auf. So legte Ehrhardt den Frauen, die häufig in mittleren und auch noch späten Schwangerschaftsstadien zur Abtreibung eingewiesen wurden, ein bis zwei Tage lang den „Metranoicter“ zur Aufdehnung des Gebärmutterhalses ein, um anschliessend die „Hysterotomia vaginalis anterior“ durchzuführen. Die erstere, sehr schmerzhafte Prozedur war - auch nach dem zeitgenössischen Stand der geburtshilflichen Operationslehren - zur Vorbereitung der nachfolgenden Operation völlig überflüssig. In weiter fortgeschrittenen Schwangerschaften war die Verwendung eines Metronoicters wegen möglicher Zerreissung der Gebärmutter lebensgefährlich. Die 23-jährige Ukrainerin Julie H. ist daran gestorben. Bei anderen „Ostarbeiterinnen“ übte Ehrhardt zum Zweck der Abtreibung den „Schuchardt-Schnitt“ aus und verletzte sie damit schwer. Der Schuchardt-Schnitt ist ein „Scheiden-Damm-Beckenboden-Schnitt durch Scheide, Vulvaring und Damm, der so tief geführt wird, dass auch die tiefe Beckenbodenmuskulatur mit gespalten wird“. ${ }^{33}$ Dieser "Hilfsschnitt bei operativen Eingriffen“ wurde sonst bei der „erweiterten vaginalen Totalexstirpation" des Uterus nach Schauta bei krebskranken Frauen angewandt. ${ }^{34}$ Ehrhardt legte den Schuchardt-Schnitt aber bei schwangeren Zwangsarbeiterinnen sowohl im Zusammenhang mit seinen fetographischen Versuchen an, als auch im Rahmen von operativen Eingriffen, in denen er einzelne Phasen jener radikalen Krebsoperationen erprobte. Auch vollzog er bei gesunden jungen Frauen etwa die „Umschneidung der Cervix“, d.h. er schnitt die Vagina in ihrem oberen Bereich um den Gebärmutterhals auf, einige Male verbunden mit der „Präparation“ eines oder beider Harnleiter (Ureter), d.h. deren operative Freilegung aus dem sie umgebenden Gewebe, dazu mindestens einmal im Zusammenhang mit der Einbringung eines Katheters in die Blase. In Hoffs Notizbuch fanden sich folgende Einträge:

„25.1.44. Vaginale sectio mit Blasenverletzung - 2 1⁄2 Stunden Operationsdauer.

25.1.44. Vaginale sectio muss auf Septisch verlegt werden wegen Fieber und Infiltrat (M.L. [Schwangerschaftsmonat] III!) [...]

28.6.44. E. macht nur vaginale sectiones bei Frühschwangerschaften! $[\ldots]$

10.7.44. Vaginale sectio bei Grav[idität] ML II. mit Umschneidung der Portio und Ureta [sic] - Präparation. Anschliessend Ureta Kathedarisierung [sic] wegen der Möglichkeit der Ureta-Verletzung bei Un-

3 PSCHYREMBEL, Klinisches Wörterbuch 1098 .

34 Josef ARTNER, Anton SCHALLER, Die Wertheimsche Radikaloperation. Anfänge, Fortschritte, Ergebnisse 1898-1968 (Wien 1968) 27.

35 StLA, LG für Strafsachen Graz 19 Vr 20/1947,175-177. Dass es sich bei den betroffenen Frauen um Zwangsarbeiterinnen gehandelt hat, lässt sich aus anderen Quellen erschliessen. terbindung des U. (Martin-Menjos) Verbrechen! Um 16 Ubr noch bei der Op[eration] “ 35

Angesichts der Verletzungen und Verstümmelungen, die der Klinikvorstand den zur Abtreibung eingelieferten „Ostarbeiterinnen“ zufügte, kam es zu Widerstandsaktionen auf der Gebärklinik mit dem Ziel, Ehrhardt wenigstens einige dieser Patientinnen zu entziehen. So stellte es jedenfalls Dr. Hoff in dem Ermittlungsverfahren gegen ihn nach 
Kriegsende dar. Als Hauptakteure dabei bezeichnete er Dr. Wolodymir Pruc und sich selbst. Sie verlegten Patientinnen in die II. Klasse (die Dr. Hoff unterstand) oder fälschten bei der Aufnahme, bei der Dr. Pruc als Dolmetscher in der Regel anwesend gewesen sein soll, die Diagnose. ${ }^{36}$ Dr. Hoffs Rettungsversuche erscheinen allerdings nicht ganz uneigennützig. Aus anderen Quellen geht hervor, dass er mit dem Klinikchef und dem reichsdeutschen Oberarzt Dr. Reinhold Elert (1909-1970) im Wettstreit um „Patientenmaterial“ lag - für Operationen der österreichischen Assistenten und, was Dr. Hoff selbst betrifft, für eigene Experimente. Am 28. Juni 1944 schrieb er in sein Notizbuch: „Gibt mir keine Fälle um meine Versuche abzuschliessen". ${ }^{37}$

Einen Beleg besonderer Art über an Zwangsarbeiterinnen vorgenommene Abtreibungen bietet ein kurzer Bericht über die Sitzung der Medizinischen Gesellschaft Steiermark vom 12. März 1944. Innerhalb dieser wissenschaftlich-professionellen Öffentlichkeit hatte Karl Ehrhardt über "Schwangerschaftsunterbrechung jenseits des 4. bis 5. Monats durch intraamniale Injektion von Wirkstoffen" vorgetragen..$^{38} \mathrm{Er}$ stellte die „Nachprüfung“ der Abtreibungsmethode eines südamerikanischen Gynäkologen vor, den „Formalin-Abort“. Der Sitzungsbericht dokumentiert allerdings nicht nur umfangreiche Versuche Ehrhardts an jungen Frauen, die unmittelbar dem Einweisungsziel Schwangerschaftsabbruch verpflichtet zu sein scheinen. Er verweist ausserdem en passant auf seinen fetographischen Forschungsschwerpunkt. Tatsächlich war Ehrhardt nicht auf der Suche nach einer „schonenden“ Abbruchsmethode, wie er vorgab. Davon kann schon angesichts seiner missbräuchlichen Krebsoperationsversuche an „Ostarbeiterinnen“ nicht die Rede sein. Die hier vorgestellte Abtreibungsmethode mit der Injektion tödlicher Gifte für die Leibesfrucht entstand vielmehr im Kontext der Fortführung seiner Forschungen an schwangeren Frauen und Tieren zur röntgenologischen Sichtbarmachung des Fötus bzw. seiner inneren Organe. Diese Tatsache belegen zum einen die anfangs zitierten Einträge aus Hoffs Notizbuch, zum anderen ein Versuchsprotokoll Ehrhardts, welches das Kriegsende in der Schreibtischschublade des Klinikvorstands überdauerte, während dieser sich längst nach Bayern abgesetzt hatte. Das maschinenschriftliche Protokoll umfasst den Zeitraum Januar bis Juni 1944 und enthält Aufzeichungen über Experimente an 85 schwangeren Frauen, zumeist Ukrainerinnen sowie einigen Russinnen und Polinnen, davon 60 im Alter zwischen 18 und 23 Jahren und 63 zum ersten Mal schwanger. Knapp zwei Drittel waren schon über den vierten Monat hinaus, vier bereits im achten Monat ihrer Schwangerschaft. Das von Ehrhardt durch Formalininjektion getötete, kurz vor seiner Geburt stehende Kind der 22-jährigen Anna H. aus Kiew war $51 \mathrm{~cm}$ lang und wog $3000 \mathrm{~g}$. Die Einträge im Versuchsprotokoll zeigen, dass er den Frauen nicht nur unterschiedliche Pharmaka zur Tötung ihrer Leibesfrüchte injizierte, sondern zudem in weit über der Hälfte der Fälle zusätzlich zu diesen Giften Kontrastmittel spritzte, um seine röntgenologischen Experimente fortzusetzen. Auch
36 Vgl. CZARNOWSKI, Material 247, 248.

37 StLA, LG für Strafsachen Graz 19 Vr 20/1947, 177.

38 Vgl. CZARNOWSKI, Material 249251. 
diese dürfte er der Medizinischen Gesellschaft Steiermark vorgestellt haben. Am 2. Juni 1944 hielt er dort einen Vortrag mit dem Titel „Beitrag zur intraamnialen Biologie und Pathologie des Kindes". ${ }^{39}$

Ehrhardts letzte in diesem Kontext entstandene Publikation erschien im Februar 1945 in der Medizinischen Zeitschrift unter der Überschrift „Atmet das Kind im Mutterleib? Ein weiterer Beitrag zur intrauterinen Biologie des Kindes “. ${ }^{40}$ In dieser Publikation kommt er dem Übergang zwischen „indizierter“ Abtreibung und ärztlichem Kindsmord so nah wie in keinem seiner vorangegangenen Texte. Er verweist auf seine bereits sechsjährige Forschungstätigkeit und man kann in seinen Ausführungen das Fortschreiten zu immer brutaleren Formulierungen und Handlungen erkennen: Unter Hinweis auf seine früheren Publikationen, in denen er gezeigt habe, „dass man durch intraamniale Injektion von kolloidalem Thorium (Thorotrast, Umbrathor) den fötalen Magen-Darmkanal und die fötale Lunge röntgenologisch sichtbar machen und so die intrauterine Trink-und Atemfunktion studieren könne" "aktive Funktionsvorgänge, die beim toten Kind natürlich [fehlten]" - formuliert er im ersten Teil seines Beitrags sein Forschungsinteresse für den „schluckenden Fötus“ folgendermaßen: „Wie liegen die Dinge beim intrauterin sterbenden Kind? Dauert die Schluckaktion bis zum Eintritt des Todes an oder erlischt sie schon vorher? Dieser Frage bin ich u.a. beim „Formalinabortus" nachgegangen" 41 - eben in jenen Experimenten, die im Versuchsprotokoll festgehalten sind und die Ehrhardt im März 1944 den Mitgliedern der medizinischen Gesellschaft Steiermark vorgestellt hatte. Der zweite Teil seines Beitrags galt erneut dem „Problem der intrauterinen Atembewegung des Kindes“, nun aber „besonders des ausgetragenen oder fast ausgetragenen Kindes". ${ }^{42}$ Die „bis jetzt“ vorliegenden Untersuchungsergebnisse seien „an Föten der ersten Schwangerschaftshälfte bzw. der Schwangerschaftsmitte gewonnen", es erhebe sich daher die Frage, ob diesen Befunden auch für die Zeit am Schwangerschaftsende Gültigkeit zukomme. Nun seien „die Möglichkeiten, unsere Studien über die intraamniale Biologie und Pathologie des Fötus auf spätere Schwangerschaftsmonate auszudehnen [...] äußerst spärlich“. Daher sei „jeder Befund, den wir gegen Ende der Schwangerschaft erheben können, von ganz besonderem Wert". Und er schreibt es dem Zufall zu („Der Zufall wollte es“), „dass ich im Laufe der vergangenen Jahre in zwei Fällen Gelegenheit hatte, die Fra-

39 StLA, LG für Strafsachen Graz, Vr $4434 / 49$, Bd 3.

40 Karl EHRHARDT, Atmet das Kind im Mutterleib? Ein weiterer Beitrag zur intrauterinen Biologie des Kindes. In: Medizinische Zeitschrift (1945) 182-183.

41 EHRHARDT, Intrauterine Biologie 182.

42 EHRHARDT, Intrauterine Biologie 183.

43 EHRHARDT, Intrauterine Biologie 183. ge der intrauterinen Atembewegungen beim ausgetragenen bzw. fast ausgetragenen Kind mit der oben geschilderten Methode röntgenologisch zu überprüfen. " 43

Eine lebendige Frucht im Leib der Frau war Voraussetzung für die Durchführung dieser „biologischen Methode“, die einen bis drei Tage dauerte, um genügend sichtbare Schatten für das Röntgenbild des Fötus zu erzeugen. Am Ende stand die Tötung der Leibesfrucht, sogar noch kurz vor der Geburt. Anders als in den vorangegangenen Publikationen gibt Ehrhardt in diesem Aufsatz keine Auskunft mehr darüber, welche schwangeren Frauen seine Versuchsobjekte waren. Abbrüche 
der Schwangerschaft aus eugenischen und gesundheitlichen Gründen waren nur bis zum 6. Monat der Schwangerschaft gestattet. Bei Abtreibungen an „Ostarbeiterinnen“ und Polinnen hingegen durfte diese Grenze auf Geheiss Contis ausdrücklich überschritten werden, wenn der operierende Arzt dazu bereit war. Ehrhardt konnte seine Experimente nur an Zwangsarbeiterinnen vollführen, die in der UFK Graz auch weit jenseits des 6. Monats zur „Interruptio“ aufgenommen wurden. Einschränkend sei jedoch darauf hingewiesen, dass ein Frankfurter Kollege, der damalige Assistenzarzt Hans Dörr, in einer Publikation von 1938 bereits erwähnt, dass Ehrhardt bei Schwangeren vom 6. bis zum 9. Monat Thorotrast durch die Bauchdecke injiziert habe. An wie vielen und welchen Patientinnen Ehrhardt diese Versuche machte, verriet Dörr nicht. ${ }^{44}$ Ehrhardt selbst hat Eingriffe bis zum 9. Monat zuvor in keinem seiner Aufsätze erwähnt, allerdings schon 1939 seine „Vermutung “ ausgesprochen, dass sich am Ende der Schwangerschaft hinsichtlich der „Atmung“ des Fötus andere Verhältnisse finden könnten als in früheren Stadien. Möglicherweise beschrieb er 1945 einen Fall aus Frankfurt und einen Fall aus Graz. Sein letzter Satz lautete lapidar: „Da die Zabl der bisher beobachteten Fälle (2) für ein abschliessendes Urteil unzureichend ist, ist die Mitteilung weiterer einschlägiger Beobachtungen erwünscht.“

\section{IV.}

Obwohl Dr. Hoff schwangere Zwangsarbeiterinnen vor den missbräuchlichen Krebsoperationsversuchen Ehrhardts zu retten suchte, führte er selbst Versuche an ihnen durch. Im Vergleich zu den Forschungen Ehrhardts sind die von Hoff als weniger eingreifend zu bezeichnen. ${ }^{45}$ Sie dauerten nicht Tage oder Wochen, sondern Stunden; die Frauen trugen keine dauernden Gesundheitsschäden davon. Sie mussten „nur“ zwei Stunden und länger „am Operationstisch“ liegen, schmerzhafte Manipulationen über sich ergehen lassen und wurden im letzten Teil des Versuchs narkotisiert. Ob dies im Zusammenhang mit dem operativen Eingriff des Schwangerschaftsabbruchs geschah oder zusätzlich, ist unbekannt. Nach der Aussage eines Zeugen hat Hoff „[...] eine Gummiblase in die Gebärmutter der Russinnen eingefübrt, die er dort stundenlang liegen liess, worauf er verschiedene Medikamente zur Anwendung brachte, und deren Wirkung durch Apparate, die mit der Blase in der Gebärmutter verbunden waren, studierte. Diese Experimente waren mit grossen Qualen für die Frauen verbunden". ${ }^{46}$ Die Apparatur entsprach der von Hermann Knaus erfundenen „Technik der Registration von Bewegungen der menschlichen Gebärmutter" 47 - die dieser allerdings nur bei nichtschwangeren Frauen angewandt hatte. Bei den injizierten „Medikamenten“ handelte es sich um das Wehenmittel Orasthin, das Narkotikum Evipan und andere. Hoff führte Versuche Bayers fort, die dieser wegen seiner Einberufung zum Militär unterbrechen musste. Das Buch erschien 1955 unter beider Namen,
44 Vgl. CZARNOWSKI, Material 255.

45 Vgl. zum folgenden ausführlich: CZARNOWSKI, Material 263-273.

46 StLA, LG für Strafsachen Graz 19 Vr 20/1947, 45Rs.

47 Hermann KNAUS, Zur Technik der Registration von Bewegungen der menschlichen Gebärmutter, Zentralblatt für Gynäkologie 57 (1933) 2658-2662. 
trug den Titel „Ovarialhormone und Uterusmotilität“ und gab darüber Auskunft, dass die „experimentelle(n) Arbeiten im Jahr 1941 begonnen wurden und bis 1945 im wesentlichen beendet" waren. ${ }^{48}$ Insgesamt wurden über 700 Untersuchungen an mehr als 600 Frauen durchgeführt. Im Vorwort wiesen die Autoren darauf hin, dass „die wesentlichen Fragen erstmalig in dieser Breite am menschlichen Uterus studiert werden konnten“ und dass es dadurch möglich sei, „die bisher unklaren und mit den Ergebnissen der Tierversuche nicht in Einklang zu bringenden Beobachtungen nach jeder Seite kritisch zu werten und so eine Darstellung der physiologischen Verhältnisse am menschlichen Uterus zu geben, in der das Tierexperiment nicht mehr Beweis der Funktion, sondern nur Bestätigung allgemein gültiger biologischer Regeln" ${ }^{49}$ sei. Ihr Forschungsinteresse galt den Bewegungen der Gebärmutter in allen ihren „Zuständen“, angefangen von den verschiedenen Phasen im natürlichen und im durch Hormonbehandlung künstlich erzeugten Menstruationszyklus, im „hormonal verwaisten Organ", während der Schwangerschaft und unter der Geburt, bis hin zur Plazentaausstossung. Dazu benutzten sie Patientinnen mit dem jeweils entsprechend vorhandenen oder operativ oder durch Hormongaben hergestellten Gebärmutterstatus, schwangere und nichtschwangere Frauen verschiedener Altersgruppen. Während Bayer offenbar vorwiegend an nichtschwangeren Frauen geforscht hatte, experimentierte Hoff an schwangeren Frauen, und zwar während der gesamten Dauer der Schwangerschaft, und "wabllos an deutschen und ausländischen", wie Hoff vor der Polizei aussagte. ${ }^{50}$ Doch von Spätschwangerschaften und Geburten abgesehen, ist davon auszugehen, dass Dr. Hoff nur an solchen Frauen experimentierte, die zum Schwangerschaftsabbruch in die Klinik gekommen waren, und das waren zwischen 1943 und 1945 hauptsächlich Zwangsarbeiterinnen. Die Art seiner Versuche hätte Schwangerschaften, die ausgetragen werden sollten, zu stark gefährdet. Aufschlüsseln nach dem Schwangerschaftsstadium lassen sich 212 Fälle. 127 Frauen waren im 1.-3. Monat, 63 im 3.-5. Monat, 21 im 5.-7. Monat und eine im 7.-8. Monat.

\section{IV.}

48 Franz HOFF, Richard BAYER, Ovarialhormone und Uterusmotilität $(=$ Beilageheft zur Zeitschrift für Geburtshilfe 144, Stuttgart 1956 [tats. 1955]), VI.

49 HOFF, BAYER, Ovarialhormone $\mathrm{V}-\mathrm{VI}$.

50 StLA, LG für Strafsachen Graz 19 $\operatorname{Vr}$ 20/1947, 137. Auch noch nach Kriegsende sprach Hoff von Deutschen, obwohl er sich auf Einheimische bezog.

51 Mündliche Auskunft Prof. Erich Burghardt, Graz, am 18. Mai 2005.
An den zwischen 1943 und 1945 zum Schwangerschaftsabbruch in die Universitätsfrauenklinik Graz eingewiesenen jungen Frauen aus Polen und Ländern der damaligen Sowjetunion wurden nicht nur politisch erzwungene Abtreibungen durchgeführt. Zusätzlich hatten sie missbräuchliche medizinische Eingriffen zu erleiden, die sich in zwei Richtungen erstreckten: eingreifende, indikationslose Operationen sowie wissenschaftliche Forschungen. Die u.a. in diesem Zusammenhang nach Kriegsende eingeleiteten Strafverfahren gegen Prof. Ehrhardt und seinen Widersacher Dr. Hoff wurden beide eingestellt. Ehrhardt hat nach 1945 nicht wieder an einer Universität gearbeitet, nahm aber weiterhin an Gynäkologenkongressen teil. ${ }^{51}$ Er betrieb zunächst eine Fach- 
arztpraxis in Frankfurt am Main, gründete später eine Privatklinik und ist 1993 im hohen Alter von 98 Jahren in Frankfurt gestorben. ${ }^{52}$ Hoff konnte seine zunächst entzogene Lehrbefugnis an der Grazer medizinischen Fakultät später zurückerlangen. Er gründete das Sanatorium Dr. Hoff in Graz-Geidorf, das vor einiger Zeit in Leech-Klinik umbenannt wurde.

\section{Abkürzungsverzeichnis}

$\begin{array}{ll}\text { BA (ehem. BDC) } & \text { Bundesarchiv Berlin (ehemals Berlin Document Center) } \\ \text { EOG } & \text { Erbgesundheitsobergericht } \\ \text { GKH } & \text { Gaukrankenhaus } \\ \text { LG } & \text { Landesgericht } \\ \text { StLA } & \text { Steiermärkisches Landesarchiv } \\ \text { UAG } & \text { Universitätsarchiv der Karl Franzens-Universität Graz } \\ \text { UFK } & \text { Universitätsfrauenklinik }\end{array}$

\section{Quellen- und Literaturverzeichnis}

Bundesarchiv Berlin, (ehem. BDC) (BA), SS-Führerakten

Steiermärkisches Landesarchiv (StLA), Frauenklinik - Gebärhaus Landesgericht für Strafsachen Graz

Universitätsarchiv der Karl Franzens-Universität Graz (UAG), Dekanatsakten der medizinischen Fakultät Personalakte Prof. Dr. Karl Ehrhardt

ARTNER, Josef, SCHALLER, Anton, Die Wertheimsche Radikaloperation. Anfänge, Fortschritte, Ergebnisse 1898-1968 (Wien 1968).

BOCK, Gisela, Zwangssterilisation im Nationalsozialismus. Studien zur Frauenpolitik und Rassenpolitik (Opladen 1986).

CZARNOWSKI, Gabriele, Vom „reichen Material ... einer wissenschaftlichen Arbeitsstätte“. Zum Problem missbräuchlicher medizinischer Praktiken an der Grazer Universitätsfrauenklinik in der Zeit des Nationalsozialismus. In: Wolfgang FREIDL, Werner SAUER (Hg.), NS-Wissenschaft als Vernichtungsinstrument. Rassenhygiene, Zwangssterilisation, Menschenversuche und NS-Euthanasie in der Steiermark (Wien 2004) 225-273.

CZARNOWSKI, Gabriele, „Die restlose Beherrschung dieser Materie“. Beziehungen zwischen Zwangssterilisation und gynäkologischer Sterilitätsforschung im Nationalsozialismus. In: Zeitschrift für Sexualforschung 14 (2001) 226-246.

CZARNOWSKI, Gabriele, Women's crimes, state crimes: abortion in Nazi Germany. In: Margaret L. ARNOT, Cornelie USBORNE (Hg.), Gender and Crime in Modern Europe (London 1999) 238-256.

CZECH, Herwig, Zwangsarbeit, Medizin und „Rassenpolitik“ in Wien: Ausländische Arbeitskräfte zwischen Ausbeutung und rassistischer Verfolgung. In: Andreas FREWER, Günther SIEDBÜRGER (Hg.), Medizin und Zwangsarbeit im Nationalsozialismus. Einsatz und Behandlung von „Ausländern“ im Gesundheitswesen (Frankfurt a.M. / New York 2004) 253-280, hier 269-273.

52 Auskunft des Standesamtes Selters/ Taunus v. 12.06.2008. 
EHRHARDT, Karl, Der trinkende Fötus. Eine röntgenologische Studie. In: Münchener medizinische Wochenschrift 84 (1937) 1699-1700.

EHRHARDT, Karl, Atmet das Kind im Mutterleib? Eine röntgenologischen Studie. In: Münchener medizinische Wochenschrift 86 (1939) 915-918.

EHRHARDT, Karl, Weitere Erfahrungen mit meiner Methode der intraamnialen Thoriuminjektion (Fetale Organographie). In: Zentralblatt für Gynäkologie 65 (1941) 114-120.

EHRHARDT, Karl, Atmet das Kind im Mutterleib? Ein weiterer Beitrag zur intrauterinen Biologie des Kindes. In: Medizinische Zeitschrift (1945) 182183.

ERBSLÖH, [Joachim], Das intrauterine Fetogramm. In: Archiv für Gynäkologie 174 (1942) 160-162. (Verhandlungen der deutschen Gesellschaft für Gynäkologie, Wien 1941).

FREIDL, Wolfgang, SAUER, Werner (Hg.), NS-Wissenschaft als Vernichtungsinstrument. Rassenhygiene, Zwangssterilisation, Menschenversuche und NS-Euthanasie in der Steiermark (Wien 2004).

FREUND, Florian, PERZ, Bertrand, Die Zahlenentwicklung der ausländischen Zwangsarbeiter und Zwangsarbeiterinnen auf dem Gebiet der Republik Österreich 1939-1945. Gutachten im Auftrag der Historikerkommission der Republik Österreich, Wissenschaftliche Redaktion: Eva BLIMLINGER (Wien 2000) (http://www.historikerkommission.gv.at/deutsch_home.html / Ergebnisberichte).

FREWER, Andreas, SIEDBÜRGER, Günther (Hg.), Medizin und Zwangsarbeit im Nationalsozialismus. Einsatz und Behandlung von „Ausländern“ im Gesundheitswesen (Frankfurt a.M. / New York 2004).

FROBENIUS, Wolfgang, Abtreibungen bei „Ostarbeiterinnen“ in Erlangen. Hochschulmediziner als Helfershelfer des NS-Regimes. In: Andreas FREWER, Günther SIEDBÜRGER (Hg.), Medizin und Zwangsarbeit im Nationalsozialismus. Einsatz und Behandlung von „Ausländern“ im Gesundheitswesen (Frankfurt a.M. / New York 2004) 283-307.

FROBENIUS, Wolfgang, Röntgenstrahlen statt Skalpell. Die Erlanger Frauenklinik und die Geschichte der Radiologie 1914-1945 (= Erlanger Forschungen Reihe B 26, Erlangen 2003).

HARTMANN, Gerhard, Im Gestern bewährt - Im Heute bereit. 100 Jahre Carolina. Zur Geschichte des Verbandskatholizismus (= Grazer Beiträge zur Theologiegeschichte und Kirchlichen Zeitgeschichte 2, Graz Wien Köln 1988).

HOFF, Franz, BAYER, Richard, Ovarialhormone und Uterusmotilität (= Beilageheft zur Zeitschrift für Geburtshilfe [und Gynäkologie] 144, Stuttgart 1956 [tats. 1955]).

POIER, Birgit, „Erbbiologisch unerwünscht“. Die Umsetzung rassenhygienisch motivierter Gesundheits- und Sozialpolitik in der Steiermark 1928-1945. In: Wolfgang FREIDL, Werner SAUER (Hg.), NS-Wissenschaft als Vernichtungsinstrument. Rassenhygiene, Zwangssterilisation, Menschenversuche und NS-Euthanasie in der Steiermark (Wien 2004) 177-224.

HAUCH, Gabriella, Zwangsarbeiterinnen und ihre Kinder: Zum Geschlecht der Zwangsarbeit. In: Christian GONSA, Gabriella HAUCH, Michael JOHN, Josef MOSER, Bertrand PERZ, Oliver RATHKOLB, Michaela C. SCHOBER, Zwangsarbeit - Sklavenarbeit: Politik-, sozial- und wirtschaftshistorische Studien (= Oliver RATHKOLB (Hg.), NS-Zwangsarbeit: Der Standort Linz der Reichswerke Hermann Göring AG Berlin, 1938-1945 1, Wien/ Köln/ Weimar 2001) 355-448.

HORNUNG, Ela, LANGTHALER, Ernst, SCHWEIZER, Sabine, Zwangsar- 
beit in der Landwirtschaft in Niederösterreich und dem nördlichen Burgenland (= Zwangsarbeit auf dem Gebiet der Republik Österreich 3, München 2004).

KARNER, Stefan, RUGGENTHALER, Peter, Zwangsarbeit in der Land- und Forstwirtschaft auf dem Gebiet Österreichs 1939-1945 (= Zwangsarbeit auf dem Gebiet der Republik Österreich 2, München 2004).

KLEINERT, Ulrike, Radium-Jubel und Röntgen-Wertheim. Gynäkologische Radiologie an der Frankfurter Universitäts-Frauenklinik von den Anfängen bis 1938 (= Frankfurter Beiträge zur Geschichte, Theorie und Ethik der Medizin 8, Hildesheim 1988).

KNAUS, Hermann, Zur Technik der Registration von Bewegungen der menschlichen Gebärmutter, Zentralblatt für Gynäkologie 57 (1933) 2658-2662.

ÖSTERREICHISCHE HISTORIKERKOMMISSION (Hg.), Zwangsarbeiter und Zwangsarbeiterinnen auf dem Gebiet der Republik Österreich 19491945. Mit Beiträgen von Mark SPOERER, Florian FREUND, Bertrand PERZ (= Zwangsarbeit auf dem Gebiet der Republik Österreich 1, München 2004).

PISCHINGER, Alfred, Über das Wesen kindlicher Atembewegungen vor der Geburt. In: Zentralblatt für Gynäkologie 65 (1941) 120-124.

PSCHYREMBEL, Willibald, Klinisches Wörterbuch, 253. Auflage (Berlin / New York 1977).

SCHEIBLECHNER, Petra, „... politisch ist er einwandfrei..." Kurzbiographien der an der Medizinischen Fakultät der Universität Graz in der Zeit von 1938 bis 1945 tätigen WissenschaftlerInnen (= Publikationen aus dem Archiv der Universität Graz 39, Graz 2002).

SCHLÜNDER, Martina, Graz 1939: Die Konsequenzen einer Fehlbesetzung. Unveröffentlichtes Manuskript, Berlin 1998.

STELZL, Barbara, Lager in Graz. Zur Unterbringung ausländischer Zivilarbeiter, Kriegsgefangener und KL-Häftlinge 1938-1945. In: Stefan KARNER (Hg.), Graz in der NS-Zeit. Begleitband zur Ausstellung (Graz 1998) 353-369. 